\title{
Roughness perception in haptic virtual reality for sighted and blind people
}

\author{
Diana Kornbrot, Paul Penn, and Helen Petrie \\ University of Hertfordshire, Hatfield, England \\ AND \\ STEPHEN FURNer AND ANDREW HARDWICK \\ BT Group Chief Technology Office
}

\begin{abstract}
Psychophysical functions for perceived roughness, relating $\ln$ (magnitude estimate of roughness) to $\ln$ (groove width), were obtained for blind and sighted participants in virtual reality using the PHANToM force feedback device. The stimuli were sinusoidal surfaces with groove widths between $0.675 \mathrm{~mm}$ and $20.700 \mathrm{~mm}$. Group functions showed a similar nonlinearity to those obtained in physical reality using rigid probes (Klatzky, Lederman, Hamilton, Grindley, \& Swendsen, 2003; Lederman, Klatzky, Hamilton, \& Ramsay, 1999). Individual functions gave a different picture. Of 23 total participants, there were 13 with wholly descending linear psychometric functions, 7 with quadratic functions similar to the group function, and 3 with anomalous functions. Individual power law exponents showed no significant effects of visual status. All analyses gave a power law exponent close to -0.80 . The implications for theories of roughness, methodologies of data analysis, and the design of haptic virtual reality interfaces are considered.
\end{abstract}

Roughness is one of the primary perceptual properties of objects. For example, Linus's attachment to his soft security blanket may evoke empathy even in the most scientific of psychophysicists. Indeed, the physical properties that determine roughness have long been of psychophysical interest. Advances in technology have added to our knowledge in several ways. There is now a wider range of wellspecified physical stimuli. Research is no longer limited to running fingers over sandpaper of different grain sizes. Nevertheless, the spacing and size of the texture elements on a surface remain the primary, if not the only, physical variables of interest. Consequently, determining the psychophysical function that relates perceived roughness to interelement spacing remains a major focus of research. Furthermore, it is now possible to produce virtual stimuli with properties that parallel those of the physical world. These developments have the potential to provide tactile interfaces for everyone, and they offer particularly exciting opportunities to improve interfaces for blind and partially sighted people. The latter is a particularly important issue, since interfaces that replace visual information with auditory and haptic information are greatly needed by blind and partially sighted people (Petrie, O'Neill, \& Colwell, 2002). Although these virtual reality devices have been developed primarily for such practical applications, they also provide potential for theoretical psychophysical investigations.

The main aim of this study was to take advantage of these advances to obtain psychophysical roughness functions in virtual reality using a force feedback device. These psycho- physical functions have implications for whether a single or a dual process underlies roughness perception; they also enable investigation of differences in virtual roughness perception between blind and sighted people, with implications for the extent to which visual experience influences touch perception. Psychophysical functions can also be used to address a range of applied issues. Differences in psychological function that result from type of probe or participants' visual status have implications for the design of tactile interfaces to many applications. A further aim of this study was to address the methodological problems that arise from differing traditions for studying roughness perception. We believe this study is unique in providing both individual analyses, in the tradition of classical psychophysics (Stevens \& Galanter, 1957), and group analyses using ANOVA techniques (Lederman \& Taylor, 1972). Because the psychophysical functions may be nonlinear, the two methods can (and do) lead to different conclusions.

Pioneering work in the psychophysical tradition used magnitude estimation to obtain the Stevens's power law exponent for roughness for individual participants. The first studies used sandpaper stimuli (Brown, 1960; Ekman, Hosman, \& Lindstrom, 1965; Marks \& Cain, 1972; Stevens \& Galanter, 1957; Stevens \& Harris, 1962; Stone, $1967)$ and found that roughness increased as granule size increased (increased granule size corresponds to increased interelement spacing, where granules are the elements). Care is needed in interpreting the term interelement spacing. For sandpaper, larger granule size always corresponds

D. Kornbrot, d.e.kornbrot@herts.ac.uk 
to greater separation between granules, meaning greater interelement spacing. Similarly, for sinusoidal stimuli, like those used here, a longer frequency corresponds to greater interelement spacing. Other work has manipulated various combinations of groove width, ridge (also known as land) width, and pattern repetition width (equal to groove width + ridge width), as is discussed below. For our sinusoidal stimuli, in which there were obviously no sharp transitions from ridge to groove, the terms groove width and interelement spacing were used interchangeably for the peak-to-peak separation that corresponded to the pattern repetition width (see Figure 2).

Other early work focused on the processes that underpin roughness discrimination rather than on the magnitude of perceived roughness. In 1924, Katz suggested a two-process model. The first process operates for fine-grained (small interelement spacing) stimuli and depends on the vibrations excited as a finger or probe is moved across a stimulus. A second process operates for coarse-grained stimuli and depends only on the spatial geometry of the stimuli (Katz, 1924/1989). Direct evidence for a two-process model comes from the work of Hollins and his colleagues (Hollins \& Risner, 2000), who found that movement, and hence vibration, are necessary for roughness discrimination for elements spaced less than $0.1 \mathrm{~mm}$ apart; above that spacing, spatial information mediated by static touch is sufficient. Roughness perception has been postulated to increase in the vibratory process and to decrease in the spatial process. Many researchers have indeed found decreasing psychophysical functions for larger interelement spacing (Bensmaïa \& Hollins, 2003; Blake, Hsiao, \& Johnson, 1997; Hollins, Faldowski, Rao, \& Young, 1993; Hollins \& Risner, 2000; Klatzky \& Lederman, 1999; Klatzky, Lederman, Hamilton, Grindley, \& Swendsen, 2003; Yoshioka, Gibb, Dorsch, Hsiao, \& Johnson, 2001).

However, we found no studies that explicitly gave the exponent for the descending portion. The present study remedies this deficiency, since the exponent is an important parameter for large interelement spacing irrespective of the transition point from small to large. The occurrence of two psychophysical functions, one ascending and one descending, might imply two processes. However, elegant physiological modeling shows how an inverted U-shaped function in evoked action potentials can be accounted for by neural codes based on firing rate variation of both excitatory and inhibitory neurons (Yoshioka et al., 2001).

Yoshioka et al. (2001) have also shown that psychophysical functions obtained from human judgments of roughness based on bare-finger exploration were extremely similar to judgments obtained from the evoked potentials of slowly adapting fibers type 1 (SA1) in the monkey cortex caused by moving a surface across the monkey's finger. The transition point to the decreasing function was about 3.2-mm interelement spacing for both the narrower fingered monkey and the wider fingered human. This suggests that the density of sensor cells in the finger, rather than finger width, is important.

Klatzky, Lederman, and their colleagues (Klatzky et al. 2003; Lederman, Klatzky, Hamilton, \& Ramsay, 1999) also investigated the transition point from ascending to descend- ing functions, using rigid probes as well as bare fingers. They showed that the transition point was lower for narrower probes than for wider ones and that the function with a wide probe was very similar to the function with a bare finger. Their model postulates that the transition, or drop, point occurs at the interelement spacing at which the probe can fully penetrate the groove. To the extent that the width of a rigid probe parallels the sensor separation in a finger, the neural code model might also predict the transition point to be lower for narrower probes. On the basis of discrimination data, the original two-process theories suggest a fixed transition point, irrespective of probe characteristics, of around $1 \mathrm{~mm}$ (Hollins \& Risner, 2000; Katz, 1924/1989).

Psychophysical judgments, by contrast, show a higher transition point - $3.2 \mathrm{~mm}$ (Hollins, Seeger, Pelli, \& Taylor, 2004) or $5 \mathrm{~mm}$ (Klatzky et al., 2003) for a bare finger. These values are nevertheless less than the average human finger width, which is about $9 \mathrm{~mm}$. Klatzky, Lederman, and their colleagues explain a transition point lower than normal finger width by deformation of the finger, plus speed of movement across the surface. Researchers who model neural firing rates suggest that the density of receptors, in combination with speed of motion, is critical in determining the transition point. However, there are as yet no data that can distinguish these two kinds of models. The PHANToM force feedback device has a virtual contact area of infinitely small proportions, equivalent to a probe of zero width. Models that rely only on the geometry of probe and surfaces theoretically predict that the psychophysical function with the PHANToM will be entirely descending. Theoretical models that include finger deformation or density of sensors do not make this prediction, since participants may grip probes differently depending on interelement spacings or probe type and thus cause changes in finger deformation or sensor contact.

The point at which a psychophysical function changes from ascending to descending is thus a key property of roughness perception. This point is undoubtedly affected by speed of movement, force exerted, friction, and whether the movement is active or passive, as well as the geometry of the surface and the probe. This study, however, focuses only on the geometry of the probe (by using both a stylus and thimble probe with the PHANToM) and the visual status of the participants (blind or sighted). The results may be compared with those obtained in physical reality with rigid probes (Klatzky \& Lederman, 1999; Klatzky et al., 2003).

Determining the transition point presents methodological problems that may not have been fully appreciated in earlier work. All of the studies cited above that showed a descending portion of the psychophysical function were based on analyses of group data. For each value on the $x$-axis, the average of all participant judgments was obtained and used as the value on the $y$-axis. The $x$-axis values were usually some form of interelement spacing (groove, ridge, or pattern width). In some cases, figures showed $\log (y)$ versus $\log (x)$ rather than $y$ versus $x$. However, in all cases, the data points were averaged across participants before the psychophysical functions were obtained. The parameters of the psychophysical function-i.e., the transition point, curvature and depth for quadratic functions, 
or the slope and intercept for linear functions-were obtained only after this averaging process.

This method of analysis is different from the original psychophysical work, which obtained individual functions for each participant (Stevens \& Galanter, 1957; Stevens \& Harris, 1962; Stone, 1967) and then gave the power law exponent as the average of the slopes obtained from the individual psychophysical functions. One advantage of the original method is that it generated the range of exponents across participants and thus gave a picture of individual diversity. Of course, if the functions were linear, then they would have no effect on the obtained mean power law exponent (as was the case for the narrow range of interelement spacing used in these early studies). An even more crucial advantage of the individual analysis method becomes apparent when the individual psychophysical functions are nonlinear. In this case, the average of the parameters of the individual function is not necessarily the same as the parameters obtained from the group function. Thus, the transition point for the group function may be substantially different from the average of the transition points of the individuals. Furthermore, a minority of participants with nonlinear individual functions may cause the group function to be nonlinear. This is misleading, as one might conclude that the function is nonlinear for every individual, when in fact it is linear for the majority of participants. Similar dangers of improper averaging have been noted for decision-bound models of categorization (Maddox, 1999), and the problem is probably quite widespread in psychology. Thus, determining whether the two methods of analysis lead to similar conclusions about roughness perception was an important aim of this study.

New technologies provide the ability to produce an ever-growing range of virtual textures and virtual haptic interfaces (Basdogan \& Srinivasan, 2002; Choi \& Tan, 2004; Drewing, Ernst, Lederman, \& Klatzky, 2004; Ellis, Ganeshan, \& Lederman, 1994; Hardwick, Furner, \& Rush, 1998; Hollins \& Risner, 2000; Hollins et al., 2004; Jansson, 1998, 2002; Jansson \& Billberger, 1999; Kitada et al., 2005; Klatzky \& Lederman, 1999; Klatzky et al., 2003; Lederman et al., 1999; McGee, Gray, \& Brewster, 2001; Moore, Broekhoven, Lederman, \& Ulug, 1991; Oakley, Adams, Brewster, \& Gray, 2002; Otaduy, Jain, Sud, \& Lin, 2004; Reed, Lederman, \& Klatzky, 1990; Sjöström, 2000; Smith, Chapman, Deslandes, Langlais, \& Thibodeau, 2001; Srinivasan, Basdogan, \& Ho, 1999; Verrillo, Bolanowski, \& McGlone, 1999). Currently, the most common type of haptic device in perception experiments is the force feedback device, like the PHANToM, for example, manufactured by SensAble Technologies (Woburn, MA). ${ }^{1}$ Figure 1 shows the PHANToM and a similar earlier device, the IE 3000. Such devices work by representing to the user the force at a single point in space. By varying the direction and magnitude of the forces exerted, a force feedback device can create a virtual object of any shape and can simulate a range of object attributes, such as hardness and roughness. Such devices can supply kinesthetic information directly to their users only in terms of the force transmitted through the probe used (typically a stylus or a thimble), whereas with physical
A

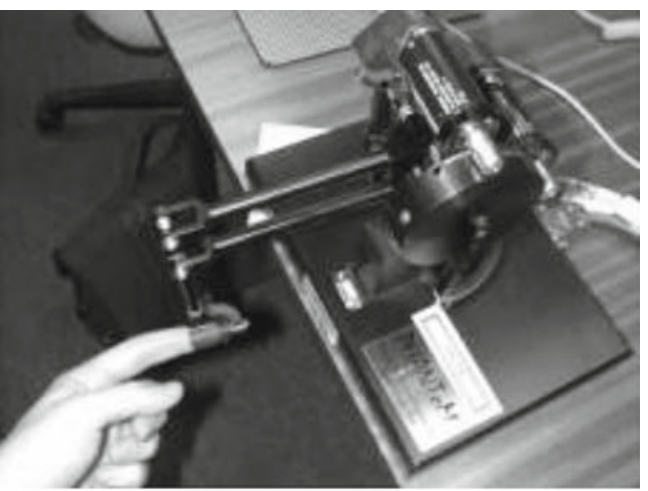

B

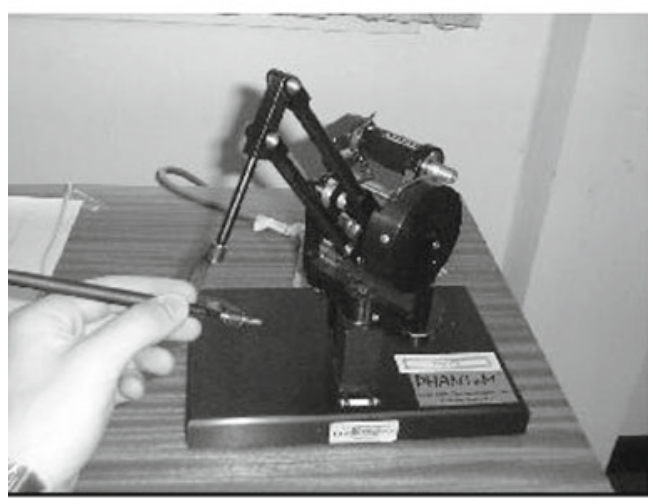

C

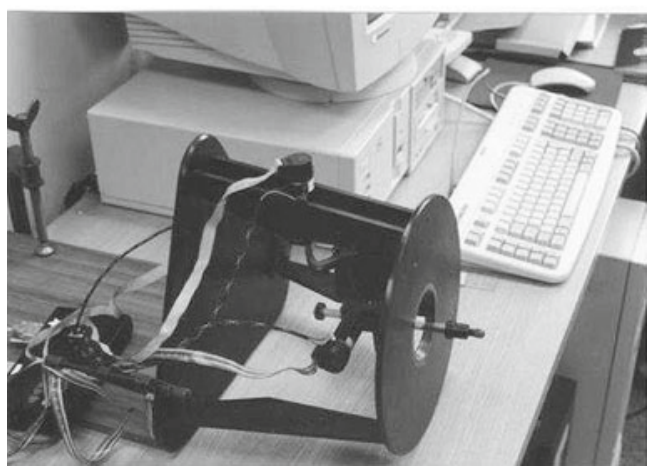

Figure 1. Force feedback devices. (A) PHANToM with thimble probe. (B) PHANToM with stylus probe. (C) IE 3000.

reality, cutaneous information is also available. Thus, to make a valid comparison between studies that use a force feedback device and those that deal with physical reality, the physical studies must use a rigid probe (Klatzky \& Lederman, 1999; Klatzky et al., 2003).

Several studies have used force feedback devices to study roughness perception in virtual reality. Jansson (1998) found that the perceived roughness of virtual textures that simulated sandpaper increased as a function of increasing interelement spacing, as it does in physical reality. Colwell, Petrie, Kornbrot, Hardwick, \& Furner (1998) used sinusoidal groove patterns and found that, for most participants, perceived roughness in virtual reality decreased with increasing interelement spacing. A study comparing the PHANToM with the IE 3000 showed slightly superior performance for the PHANToM (Weisenberger, Krier, \& Rinker, 2000). Studies using the PHANToM and regu- 
larly spaced elements all found that perceived roughness decreased with increased interelement spacing (Drewing et al., 2004; McGee et al., 2001; Wall \& Harwin, 2001). The present study used sinusoidal stimuli, because of the possibility that tactile space, like visual space, might have been successfully modeled as a Fourier decomposition of spatial frequencies. Sinusoidal stimuli are difficult to produce in physical reality, providing a further reason for investigating such stimuli in virtual reality.

Force feedback devices may have probes of different widths corresponding to the rigid links in physical reality. The PHANToM, as used in the present study, has a wide thimble probe and a narrow stylus probe. When these two types of probes are handled freely (i.e., not connected to any computer), they may be experienced by all participants (and seen by sighted participants) as different (see Figure 1). However, both probes, as used in our research, simulate interactions at a single point. Nevertheless, because participants knew that the probes were different, they may have generated differences in one or more parameters relating to an individual's interaction with a virtual texture. The perceptions generated by different force feedback devices may also differ due to the algorithms the devices use or their physical properties. To this end, the stimuli and methodology that Colwell et al. (1998) used with the IE 3000 were replicated here to allow precise comparisons.

The effect of visual status on the perception of roughness with the PHANToM was investigated for both theoretical and practical reasons. Theoretically, it might be hypothesized that blind participants, due to their greater use of the haptic sense, would have more sensitive roughness perception than sighted participants. Conversely, it might be hypothesized that sighted participants would be more sensitive, since they also have visual information to supplement their haptic perception. Differences in sensitivity might then lead to differences in psychophysical function. A finding of no effect of visual status would support the autonomy of the haptic sense. On a practical level, haptic devices have the potential to enhance access to computerbased information, particularly for blind people. In order to ensure accessibility, it is essential that designers know of any relevant differences between blind and sighted people.

In summary, the present study investigated the effect of visual status and probe type in virtual reality on the form of the psychophysical function relating $\ln$ (magnitude estimate of roughness) to $\ln$ (interelement spacing).

\section{METHOD}

\section{Participants}

There were 23 participants, 13 sighted and 10 blind. The sighted participants comprised 6 males and 7 females, with ages ranging from 19 to 36 years, all of whom were university students with no reported sensory or motor impairments. The blind participants comprised 8 males and 2 females, with ages ranging from 19 to 54 years. These participants had no useful vision but reported having no other sensory or motor impairments. Of these, 5 were blind from birth or early blind (before 2 years of age), and the remaining 5 lost their sight between the ages of 8 and 42 (late blind); see Table 1 for details. All the blind participants were volunteers recruited from a panel of blind and partially sighted individuals who participate in research at the Sensory Disabilities Research Unit at the University of Hertfordshire.

\section{Apparatus}

The PHANToM 1.0 device was controlled by a Pentium II $400 \mathrm{MHz}$ computer with $64 \mathrm{MB}$ RAM running Windows NT. A set of Sanyo PH $200 \mathrm{~N}$ headphones played white noise to the participants for the duration of the experiment. The intensity of the white noise was adjusted so that no participant could detect any auditory cues from the PHANToM.

\section{Stimuli}

The stimuli consisted of the same 10 sinusoidal virtual textures used by Colwell et al. (1998), developed with the same haptic rendering software (Hardwick et al., 1998). The algorithm is sufficiently robust that the probe's speed of motion does not change the presented texture and thus cannot affect the results. Using a coordinate system in which $x$ is horizontal to the right, $y$ is horizontal away from the user, and $z$ is vertical, the textured surface is in the $x-y$ plane. ${ }^{2}$ The sinusoidal ridges and troughs are in the $z$ direction and run parallel to the $y$-axis. Figure 2 illustrates the parameters of the sinusoidal waveform. The amplitude of the sinusoidal grooves was constant across the virtual textures at $0.1125 \mathrm{~mm}$ in the $z$ direction. The sinusoidal grooves increased from 0.675 to $20.700 \mathrm{~mm}$ in width in 10 equal increments of $0.225 \mathrm{~mm}$. The groove width for these stimuli thus represented interelement spacing. The groove width of $1.575 \mathrm{~mm}$ was used as the standard texture for the magnitude estimation comparisons. The textured space ran from $x=-20 \mathrm{~mm}$ to $x=+20 \mathrm{~mm}$. There are smooth portions of $15 \mathrm{~mm}$ at each edgethat is, from $x=-35 \mathrm{~mm}$ to $x=-20 \mathrm{~mm}$ at the left edge and from $x=+20 \mathrm{~mm}$ to $x=+35 \mathrm{~mm}$ at the right edge. The extent of the grooves is effectively infinite in the $y$ direction. The participant was seated squarely facing the desk that held the PHANToM, and the seat was adjusted so that the bottom of the texture, corresponding to $z=-0.0563 \mathrm{~mm}$, appeared to be on the desk. Participants were not given any visual representations of the virtual textures.

\section{Design}

The task was estimation of magnitude of roughness. Participants first assigned a modulus number to the standard stimulus and then gave a number that expressed the ratio of the perceived roughness of a stimulus presented by the PHANToM relative to the modulus number. The estimate of magnitude of roughness was the dependent variable. All participants completed 6 runs of the 10 virtual textures with varying groove widths and with two different probes (stylus or thimble), making 12 runs in all. Visual status with two levels, blind and sighted, was investigated as a between-groups factor. The experiment thus contained three repeated-measures factors: probe with 2 levels, stylus and thimble; groove width with 10 levels; and run number with 6 levels. Each participant made 120 judgments (10 textures $\times 6$ runs $\times 2$ probes). A run comprised one presentation of each of the 10 groove widths, with a single probe, in random order. Participants performed 6 runs with one probe followed by 6 runs with the other probe, with the order of the probe type counterbalanced among participants.

\section{Procedure}

Sighted participants were blindfolded for the duration of the experiment to prevent them from picking up any cues as to the dimensions of the virtual textures by visually monitoring the movement of their hand. Each participant then donned the headphones and was seated in front of a desk holding the PHANToM. The seat was adjusted so that the textured surface appeared to be lying on the desk. Participants were informed that the white noise was necessary to drown out cues from the motor. They were then instructed to feel the virtual texture by sweeping the stylus or thimble probe across each virtual texture once from left to right starting in the flat portion $15 \mathrm{~mm}$ to the left of the beginning of the texture; they were encouraged to use the same force and speed for each sweep, although this was not controlled. Next, participants were presented with the standard stimulus and instructed to judge its roughness and give it a number of their own choice, the modulus. The magnitude estima- 
Table 1

Individual Power Law Exponents From Linear Regressions and Associated Levels of Significance and Adjusted $\boldsymbol{r}^{2}$ As a Function of Visual Status and Probe

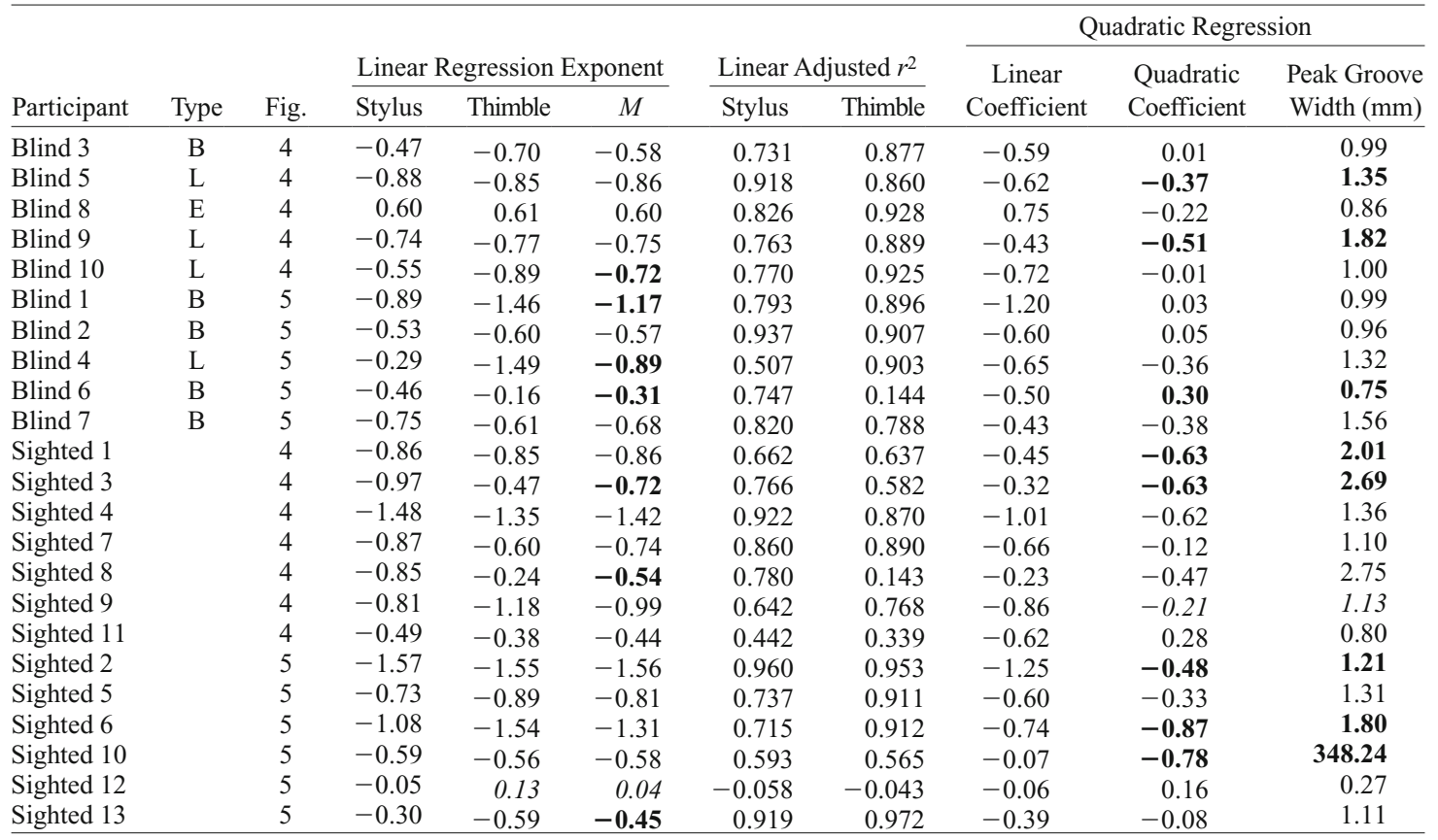

Note-B, blind from birth; E, early blind (before 2 years of age); L, late blind (after 2 years of age). In the first three columns of numbers, numbers in italics indicate no significant effect of $\ln$ (groove width), and numbers in boldface indicate that exponents for thimble and stylus were significantly different. In the last three columns of numbers, numbers in boldface indicate that the quadratic term in the ANCOVA was significant for both probes, whereas numbers in italics indicate that it was significant for only one probe.

tion procedure was explained, and participants were instructed to give each subsequent stimulus a number representing the ratio of the roughness of that stimulus to that of the standard. Examples were given relative to the participant's own chosen modulus. For example, if a participant chose 20 as the modulus, then the participant would be told, "if the stimulus appears twice as rough as the standard, call it 40 ; if it appears half as rough, call it 10." This procedure allowed participants to choose their own range of numbers but aimed to ensure that judgments were ratios. Participants then completed the 12 runs in counterbalanced order. There was a short break between runs.

\section{RESULTS}

The raw dependent variable for all analyses is $\ln$ (magnitude estimate). Magnitude estimate (ME) is the ratio of the number given by the participant divided by the modulus number the participant had assigned to the standard stimulus (i.e., judgments were normalized).

\section{Group Psychophysical Functions}

Magnitude estimation functions for blind and sighted participants with stylus and thimble probes are shown in Figure 3. In the ANOVA tradition, all observations for a given value of visual status, probe type, and groove width are first logged and then averaged The slopes of the four functions shown in Figure 3 are given as the power law exponents for each of the four possible combinations of visual status and probe. Only participants whose individual psychophysical functions all had significant negative exponents are included in the group analyses reported here. Data from 2 participants were excluded: Blind Participant 8 , who had significant positive exponents for stylus and thimble (see Table 1 and Figure 4), and Sighted Participant 12, who had completely flat functions for both probes (see Table 1 and Figure 5). However, analyses that included all participants' data gave similar findings but with reduced power due to the wider range of individual differences. Best-fitting linear functions are superimposed for the four functions. It appears that the linear function for the blind participants using a stylus has a shallower slope, and hence a smaller power law exponent, than the other three functions. The interactions in the ANCOVA described below show that this difference in slope is statistically significant. A post hoc comparison of the slope of the blind stylus condition compared with the other three conditions was also significant. Figure 3 also suggests systematic nonlinearity.

In order to test whether the differences in slopes apparent in Figure 3 were statistically significant, an ANCOVA was conducted. The dependent variable was mean (over participants) $\ln (\mathrm{ME})$. Visual status was a between-groups factor, and probe was a within-groups factor. Groove width (ln) was entered as a metric, but independent, covariate in the

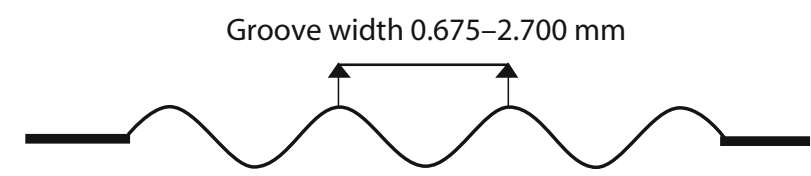

Amplitude (i.e., half base to peak height) $0.1125 \mathrm{~mm}$

Figure 20. Parameters of the sinusoidal waveform. 


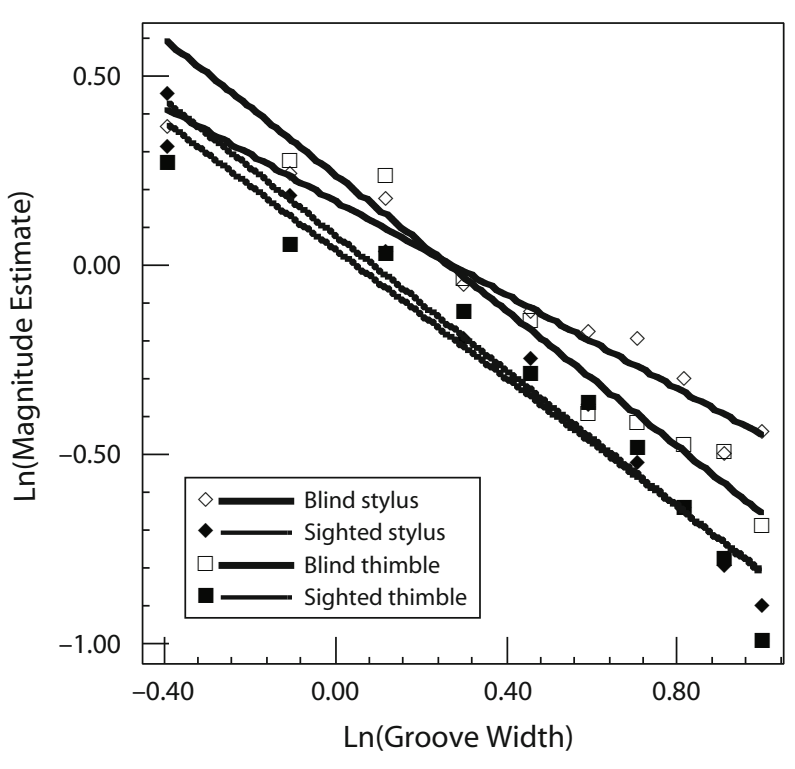

Figure 3. Group psychophysical functions for blind and sighted participants with stylus and thimble probes. (Data from participants with psychophysical functions that did not have significant negative exponents were excluded.)

analysis. This method ensured that the true magnitude of the $\ln$ (groove width) was taken into account. No (incorrect) assumption as to equal spacing of $\ln$ (groove width) was made, as would be the case if $\ln$ (groove width) were entered as a within-groups factor and a trend analysis conducted. There is no implication that $\ln$ (groove width) is a covariate in the sense of being a correlational variable, which the analysis aims to control for statistically due to lack of experimental control. $\operatorname{Ln}$ (groove width) was manipulated experimentally and hence was an independent metric variable. In such an analysis, significant differences in slope are indicated by significant interactions between factor variables and the $\ln$ (groove width) covariate. The ANCOVA gave a significant main effect for visual status $[F(1,32)=55.4, p<$ .0005 , partial $\left.\eta^{2}=.63\right]$ and $\ln$ (groove width) $[F(1,32)=$ $740.8, p<.0005$, partial $\left.\eta^{2}=.96\right]$. The main effect of visual status of course had no effect on the power law exponent. There were also significant interaction effects of $\ln ($ groove width $) \times$ visual status $[F(1,32)=5.7, p=.023$, partial $\left.\eta^{2}=.15\right]$ and $\ln ($ groove width $) \times$ visual status $\times$ probe $\left[F(1,32)=4.6, p=.040\right.$, partial $\left.\eta^{2}=.13\right]$. These interactions correspond to the significantly shallower slope of the function for blind participants using a stylus compared with the other three functions, as shown in Figure 3.

In order to test for quadratic effects in the functions in Figure 3, a similar ANCOVA that also included a quadratic term $[\ln (\text { groove width })]^{2}$ was conducted. Type 1 sums of squares were used so that a significant quadratic term was calculated over and above any linear effect. The quadratic term was statistically significant at the $95 \%$ confidence level $[F(1,28)=7.1, p=.013]$. The quadratic function peaked at a groove width of $1.25 \mathrm{~mm}, 95 \%$ confidence level with limits $1.12,1.39$. Although the quadratic term is significant at the $95 \%$ confidence level, it increased the adjusted $r^{2}$ for the full model only from .954 to .977 . At a more stringent confidence level of $99 \%$, neither the slope differences nor the quadratic terms were statistically significant.

These group analyses, taking means across participants, are included for purposes of comparison with other studies. In fact, a more informative and appropriate ANCOVA analysis would include participant as a random effect, with probe nested under participant. Such an ANCOVA analysis including only a linear term for $\ln$ (groove width) gave a significant effect for $\ln$ (groove width) only $[F(1,410)=$ $260.6, p<.0001]$. That is, the shallower slope for the blind stylus condition was no longer statistically significantly different from the slopes for the other three functions. The mean exponent was 0.80 . The confidence limits for the analysis including participants were $.70-.90$, tighter than the limits of .65-.95 for the analysis not including participants. The parallel ANCOVA including a quadratic term still gave a significant quadratic effect $[F(1,406)=5.6$, $p=.019]$. The quadratic term made a minimal improvement in adjusted $r^{2}$, from .433 to .443 . (The adjusted $r^{2}$ was much smaller, as there was then substantial betweenparticipants variance that had not been glossed over.)

In summary, the mean power law exponent was -0.80 , with $95 \%$ confidence levels of .70-.90. Group analyses of the functions in Figure 3 show a slightly lower slope for the blind stylus function. However, the effect size $\left(\eta^{2}\right)$ was small - on the order of .15-relative to the effect size for $\ln$ (groove width), which was .96, and $\eta^{2}$ was significant only at the $95 \%$, not the $99 \%$, level. This effect disappeared when participant was included as a random effect. Quadratic effects, although present, were small and not significant at the $99 \%$ level of significance. A deeper look at psychophysical functions requires analysis at the individual level.

\section{Individual Psychophysical Functions: General}

Individual psychophysical functions were obtained for all participants separately for thimble and stylus. These functions (over six replications) of mean $\ln (\mathrm{ME})$ as a function of $\ln$ (groove width) are shown in Figures 4 and 5. For each participant, an ANCOVA was conducted, including all possible interactions, with $\ln (\mathrm{ME})$ as the dependent variable, probe as a repeated measures factor with levels stylus and thimble, and $\ln$ (groove width) and $\ln$ (groove width) ${ }^{2}$ (quadratic term) as metric covariates. The main effects of probe, indicating overall different means for stylus and thimble, were not statistically significant for 12 participants (5 blind, 7 sighted). That is, all participants whose data are shown in Figure 4 had no significant difference in the height of the stylus and thimble functions; they showed very similar functions for these variables, as Figure 4 illustrates. The data for the other 11 participants, who had a significant effect of probe, are shown in Figure 5. Of these, 3 blind and 3 sighted participants showed functions with stylus significantly greater than thimble, and 2 blind and 3 sighted participants had functions with thimble significantly greater than stylus.

\section{Individual Psychophysical Functions: \\ Power Law Exponents}

Stevens's power law exponents were obtained for all participants as the slope of the linear regression of $\ln (\mathrm{ME})$ 

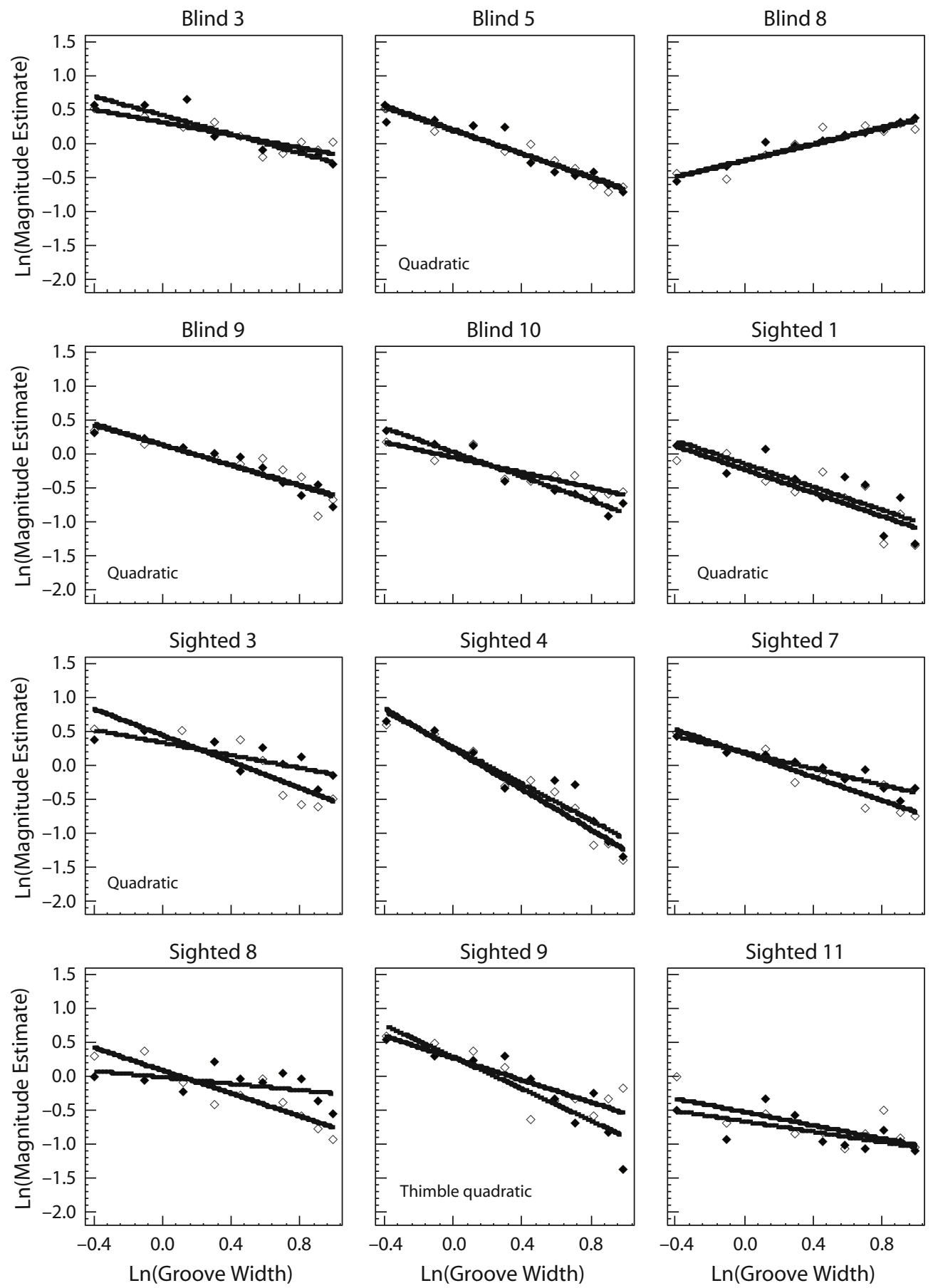

Figure 4. Fitted magnitude estimation functions for participants with highly similar thimble and stylus functions. Open triangles represent the stylus; filled circles, the thimble.

on $\ln$ (groove width) separately for the stylus and thimble conditions. The obtained exponents are shown in Table 1 together with their associated adjusted $r^{2}$ and the visual impairment type of the blind participants. Twenty-one out of 23 participants ( 9 out of 10 blind, 12 out of 13 sighted) had significant negative exponents. Blind Participant 8 , who was early blind (Figure 4), had highly significant and nearly identical positive exponents for both stylus and thimble. Sighted Participant 12 (Figure 5) showed no effect of groove width, but estimates with the stylus were substantially greater in roughness than those with the thimble. The 7 participants with significantly different exponents for the two probe types are indicated in bold in Table 1. Of these participants, 4 had more negative exponents for thimble than stylus (Blind Participant 1, 4, and 10, and Sighted Participant 13), and 3 had more nega- 
tive exponents for stylus than thimble (Blind Participant 6 and Sighted Participant 3 and 8).

In order to investigate the effects of visual status (between groups) and probe (within group) on the psychophysical function, an ANOVA was conducted with Stevens's power law exponent as the dependent variable. Data from the two participants who did not have a significant negative exponent were excluded. The mean Stevens's power law exponent was -0.81 , with $95 \%$ confidence limits $-0.69,-0.93$.
There were no statistically significant effects for visual status $[F(1,19)=.93, p=.347]$ or for probe with a multivariate $[F(1,19)=1.17, p=.293]$ or for the interaction between probe and visual status $[F(1,19)=20.21, p=.154]$.

The power law exponents obtained with the PHANToM were compared with those found with the IE 3000 (Colwell et al., 1998), as shown in Table 20. For the IE 3000, 9 out of 22 participants gave significant negative exponents compared with 21 out of 23 for the PHANToM. A test of this
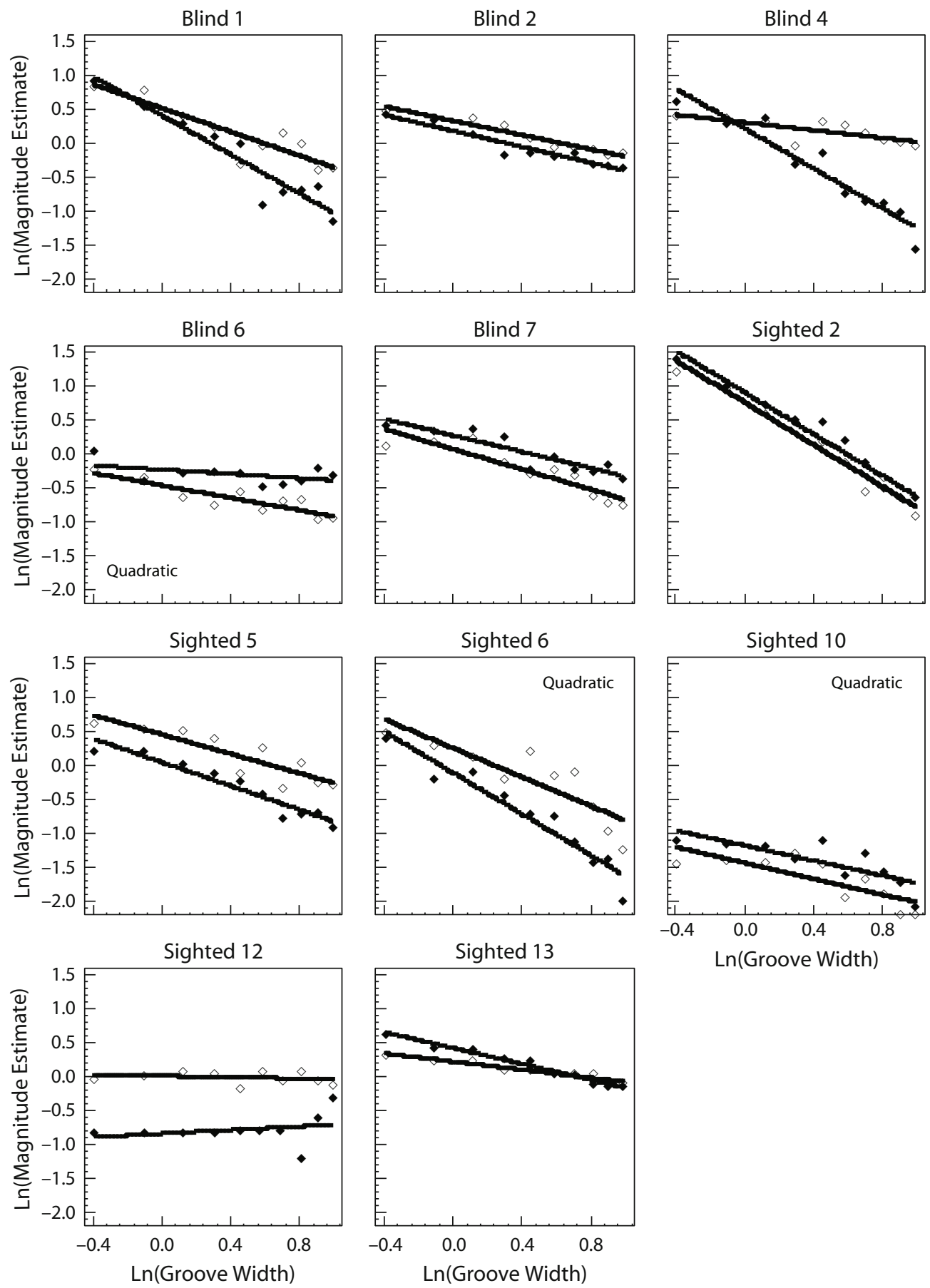

Figure 5. Fitted magnitude estimation functions for participants with less similar thimble and stylus functions. Open triangles represent the stylus; filled circles, the thimble. 
Table 2

Incidence of Significant Negative, Nonsignificant, and Significant Positive Exponents for Sighted and Blind Participants for the IE 3000 As Reported in Colwell et al. (1998) and for the PHANToM

\begin{tabular}{lcccccccc}
\hline & \multicolumn{3}{c}{ IE 3000} & & \multicolumn{3}{c}{ PHANToM } \\
\cline { 2 - 4 } Visual Status & $\begin{array}{c}\text { No. Sig. Neg. } \\
\text { Exponents }\end{array}$ & $\begin{array}{c}\text { No. Nonsig. } \\
\text { Exponents }\end{array}$ & $\begin{array}{c}\text { No. Sig. Pos. } \\
\text { Exponents }\end{array}$ & & $\begin{array}{c}\text { No. Sig. Neg. } \\
\text { Exponents }\end{array}$ & $\begin{array}{c}\text { No. Nonsig. } \\
\text { Exponents }\end{array}$ & $\begin{array}{c}\text { No. Sig. Pos. } \\
\text { Exponents }\end{array}$ \\
\hline Blind & 4 & 1 & 4 & & 9 & 0 & 1 \\
Sighted & 5 & 6 & 2 & & 12 & 1 & 1 \\
All participants & 9 & 7 & 6 & & 21 & 1 & 1 \\
\hline
\end{tabular}

comparison gave a significant difference $\left(c^{2}=120.9, p=\right.$ $.0004)$.

\section{Individual Psychophysical Functions: Transitions From Ascending to Descending}

The form of the psychophysical functions was examined using the coefficients in the quadratic ANCOVA conducted for each participant. Since only 1 participant (Sighted Participant 9) had a significant interaction between probe and $\left[\ln (\text { groove width) }]^{2}\right.$, simpler individual ANCOVAs were conducted with just ln(groove width) and $\left[\ln (\text { groove width) }]^{2}\right.$ as explanatory variables (i.e., excluding probe). The rightmost columns of Table 1 give the linear and quadratic coefficients and peak groove width for these regressions, with significant quadratic terms indicated in bold [peak groove width $=$ - quadratic coefficient/2(linear coefficient)].

Thirteen out of 23 participants had a wholly descending psychometric function, indicated by a significant linear trend with negative slope and no significant quadratic trend. The mean exponent for these participants alone was -0.79 (confidence limits: $-0.62, .95$ ). Seven participants (Blind Participant 5, 6, and 9, and Sighted Participant 1, 2,3 , and 6) showed a rising psychometric function for lower groove widths and a descending function for higher groove widths. The transition points as measured by peak groove width ranged from $0.78 \mathrm{~mm}$ to $1.82 \mathrm{~mm}$. Three participants had anomalous results: Blind Participant 8 had a wholly ascending function; Sighted Participant 12 had a flat function with no significant linear or quadratic trend; Sighted Participant 10 had a noisy function with only the quadratic term significant, although the simple linear analysis did give a significant negative exponent.

\section{DISCUSSION}

\section{Power Law Exponents From Psychophysical Functions}

The power law exponent was close to a value of -0.80 , whichever analysis method was used. Group ANOVA, including participants, gave exponent of -0.80 (confidence limits: $-0.70,-0.90$ ) (excluding 2 anomalous participants); the mean of individual exponents from linear analyses for these same participants was $-0.81(0.68$, $0.92)$; mean individual exponents for the 13 participants with wholly negative functions was $0.79(0.62,-0.95)$. Thus, one can conclude that for large interelement spacing, the Stevens's power law exponent for roughness is about -0.80 in both virtual and physical reality. The functions obtained by Johnson and his coworkers (Connor,
Hsiao, Phillips, \& Johnson, 1990; Johnson \& Hsiao, 1992; Yoshioka et al., 2001) and of Klatzky and Lederman's group (Klatzky \& Lederman, 1999; Klatzky et al., 2003) would be consistent with an exponent of -0.80 , although they did not explicitly give an exponent. It is interesting that the exponent for small interelement spacing is close to +0.80 (Hollins et al., 2004; Stevens \& Galanter, 1957; Stevens \& Harris, 1962; Stone, 1967).

\section{Form of the Psychophysical Function}

Conclusions about the form of the psychophysical function depend on the method of analysis. However, as noted above, all analyses give descending functions for large interelement spacing. This supports either the neural coding model or a separate, spatially based process for larger interelement separations. These are difficult to distinguish experimentally, although the single process is more parsimonious and has physiological support (Yoshioka et al., 2001).

Analyses based on group data are best fit by a quadratic psychophysical function with a peak at an interelement spacing of $1.25 \mathrm{~mm}$. The value of $1.25 \mathrm{~mm}$ was obtained by combining data over group and probe, as these factors had low effect sizes and were not significant at the $99 \%$ confidence level. This does not support the prediction that the zero contact point of the PHANToM would lead to a wholly descending psychophysical function. Instead, it suggests that the transition point is not completely determined by the width of the probe relative to groove width. The functions in Figure 3 are in fact very similar to those obtained for a 2-mm probe in physical reality (Klatzky \& Lederman, 1999; Klatzky et al., 2003). Thus, the group data provide no reason to suppose any differences between virtual and physical reality with a narrow rigid probe, even though the virtual probe effectively has zero width.

The individual analyses tell a different story. The psychophysical functions in Figures 4 and 5 graphically demonstrate the range of roughness perception patterns across different individuals. At the individual level, 13 participants (more than half the sample) had linear psychophysical functions for the complete range of stimuli from 0.675 to $20.700 \mathrm{~mm}$. For all of these participants, the transition from an increasing to a decreasing psychophysical function had to be substantially below the peak of $1.25 \mathrm{~mm}$ found for our group quadratic function and below most of the group function peaks obtained by previous researchers (Klatzky \& Lederman, 1999; Klatzky et al., 2003). Unfortunately, the reliability of the PHANToM for groove widths lower than $0.675 \mathrm{~mm}$ was low, so we could not test behavior at lower grooved widths. Seven participants had significant quadratic 
trends with peaks at groove widths within the stimulus range (minimum $0.75 \mathrm{~mm}$, maximum $20.69 \mathrm{~mm}$ ). These 7 did show behavior that was broadly consistent with the group data. Finally, 3 participants had anomalous results. This wide range of individual functions may also occur in physical reality with a rigid probe. For example, previous researchers (Klatzky \& Lederman, 1999; Klatzky et al., 2003) reported rejecting some participants because of anomalous judgments.

A substantial minority of participants did have quadratic functions with transition points similar to those in physical reality, even with a zero-width probe. Consequently, a probe narrower than the groove width is not sufficient to ensure a wholly descending psychophysical function for all people. This implies that geometry alone cannot account for roughness perception. Using the zero contact width probe provides evidence for factors other than geometry that could not be obtained from physical reality experiments. Nevertheless, the majority of participants showed wholly negative functions. So for some people, the transition to a negative function may indeed be wholly determined by whether the probe can penetrate the groove. The diversity of individual roughness perception using rigid probes is itself an important finding.

\section{Methodological Issues}

This study provides a clear illustration of the dangers of ignoring individual analyses. The group analyses suggest a quadratic psychometric function with peak groove width of $1.25 \mathrm{~mm}$. Nevertheless, more than half of the participants had wholly descending linear psychometric functions.

\section{Haptic Perception by Blind and Sighted People}

There were no significant differences between the blind and sighted participants. A wide range of behaviors was apparent for both blind and sighted participants. In particular, both the blind and the sighted groups included some participants with a pattern showing quadratic effects and others without it. Both groups included some participants who showed a main effect of probe (Figure 5) and others who did not (Figure 4). Finally, both groups included some participants who showed differences in exponents for stylus and thimble and others who did not.

\section{Devices and Probes}

There were no consistent differences between the psychometric functions for the stylus and thimble probes, although a few participants did have statistically significant different functions. Our results are similar to those obtained by Colwell et al. (1998) with the IE 3000 device, using an identical procedure. Hence the findings are likely to be generalizable to other 3-D force feedback devices. More of the participants showed significant effects of groove width with the PHANToM, implying that it is more reliable.

\section{The Potential for Virtual Haptic Reality}

The finding that most people, both blind and sighted, can reliably discriminate virtual textures in terms of roughness using force feedback devices augurs well for the potential of these devices. They might be used to sub- stitute for the symbolic use of color or shading in virtual interfaces for blind people or to enhance such interfaces for sighted people. The first caveat is that a minority of people would not be able to use such devices effectively. The second caveat is that there are likely to be individual preferences for different force feedback probes, just as there are for different pointing devices such as mouse, tracker ball, or joystick.

\section{Summary}

The psychometric function for roughness in virtual reality for large interelement spacing is a power law with exponent -0.80 . This result is consistent with results from other studies in both physical and virtual reality. The form of the psychometric function with a zero contact point device had both ascending and descending portions for a substantial minority ( 7 out of 23) of participants. This implies that penetration of the groove width is not sufficient for a descending psychometric function.

Individual psychometric functions showed considerable diversity. The majority of participants (13 out of 23) had functions that were wholly linearly descending. In spite of this diversity, there was no effect of either visual status or probe. This diversity has implications for virtual reality applications. Conclusions based on individual analyses are not the same as those based on grouped analyses. In our view, this difference suggests that individual analyses should always be conducted and should precede any group analyses.

At a theoretical level, any satisfactory account of the mechanisms underlying haptic perception must account for these functions, as well as for those already obtained in virtual and physical reality with stimuli of quite diverse geometric forms. Even in physical reality, much remains to be learned about the form and determinants of the psychophysical function.

\section{AUTHOR NOTE}

The research was supported by a collaborative grant from the Economic and Social Research Council and British Telecommunications to H.P. The authors gratefully acknowledge the assistance of all the blind and sighted individuals who took part in these studies. Thanks also to three anonymous referees for their constructive comments. P.P. is now at the School of Psychology, University of East London. H.P. is now at the Department of Computer Science, University of York. This article is based in part on a dissertation submitted by P.P. to the University of Hertfordshire in partial fulfillment of the $\mathrm{PhD}$ requirements. Correspondence concerning this article should be addressed to D. Kornbrot, School of Psychology, University of Hertfordshire, College Lane, Hatfield, AL10 9AB, England (e-mail: d.e.kornbrot@herts.ac.uk).

\section{REFERENCES}

Basdogan, C., \& Srinivasan, M. A. (2002). Haptic rendering in virtual environments. In K. M. Stanney (Ed.), Handbook of virtual environments: Design, implementation, and applications (pp. 117-134). Mahwah, NJ: Erlbaum

Bensmaïa, S. J., \& Hollins, M. (2003). The vibrations of texture. Somatosensory \& Motor Research, 20, 33-43.

Blake, D. T., Hsiao, S. S., \& Johnson, K. O. (1997). Neural coding mechanisms in tactile pattern recognition: The relative contributions of slowly and rapidly adapting mechanoreceptors to perceived roughness. Journal of Neuroscience, 17, 7480-7489.

Brown, I. D. (1960). Visual and tactual judgments of surface roughness. Ergonomics, 3, 51-61. 
CHOI, S., \& TAN, H. Z. (2004). Toward realistic rendering of haptic textures. IEEE Computer Graphics \& Applications, 2, 40-47.

Colwell, C., Petrie, H., Kornbrot, D., Hardwick, A., \& Furner, S. (1998). The use of haptic virtual environments by blind people. In D. Sharkey, D. Rose, \& J. -I. Lindström (Eds.), Proceedings of the Second European Conference on Disability, Virtual Reality, and Associated Technologies, Skövde, Sweden. Available at www.icdvrat.reading .ac.uk/1998/papers/1998_13.pdf.

Connor, C. E., Hsiao, S. S., Phillips, J. R., \& Johnson, K. O. (1990). Tactile roughness-Neural codes that account for psychophysical magnitude estimates. Journal of Neuroscience, 10, 3823-3836.

Drewing, K., Ernst, M. O., Lederman, S. J., \& Klatzky, R. L. (2004). Roughness and spatial density judgments on visual and haptic textures using virtual reality. Paper presented at the 4th International Conference EuroHaptics, Munich.

Ekman, G., Hosman, J., \& Lindstrom, B. (1965). Roughness, smoothness, and preference: A study of quantitative relations in individual subjects. Journal of Experimental Psychology, 70, 18-26.

Ellis, R. E., Ganeshan, S. R., \& Lederman, S. J. (1994). A tactile sensor based on thin-plate deformation. Robotica, 12, 343-351.

Haptics Community (2004). Retrieved August 10, 2006 from haptic .mech.northwestern.edu/intro/gallery/.

Hardwick, A., FURNER, S., \& RUSH, J. (1998). Tactile display of virtual reality from the World-Wide Web-A potential access method for blind people. Displays, 18, 153-161.

Hollins, M., FaldowsKi, R., RaO, S., \& Young, F. (1993). Perceptual dimensions of tactile surface texture: A multidimensional-scaling analysis. Perception \& Psychophysics, 54, 697-705.

Hollins, M., \& Risner, S. R. (2000). Evidence for the duplex theory of tactile texture perception. Perception \& Psychophysics, 62, 695-705.

Hollins, M., Seeger, A., Pelli, G., \& Taylor, R. (2004). Haptic perception of virtual surfaces: Scaling subjective qualities and interstimulus differences. Perception, 33, 1001-1019.

JANSSON, G. (1998). Can a haptic force feedback display provide visually impaired people with useful information about texture roughness and 3-D form of virtual objects? Paper presented at the Second European Conference on Disability, Virtual Reality and Associated Technologies, Skövde, Sweden.

JANSSON, G. (2002). Perceiving complex virtual scenes without visual guidance. In M. L. McLaughlin, J. P. Hespanha, \& G. S. Sukhatme (Eds.), Touch in virtual environments: Haptics and the design of interactive systems (pp. 169-179). Upper Saddle River, NJ: Prentice Hall.

Jansson, G., \& Billberger, K. (1999). The PHANToM used without visual guidance. Paper presented at the First PHANToM Users Research Symposium, Heidelberg, Germany.

Johnson, K. O., \& HsiaO, S. S. (1992). Neural mechanisms of tactual form and texture perception. Annual Review of Neuroscience, 15, 227-250.

Katz, D. (1989). The world of touch (L. Krueger, Trans.). Hillsdale, NJ: Erlbaum. (Original work published 1924)

Kitada, R., Hashimoto, T., Kochiyama, T., Kito, T., OKada, T., Matsumura, M., ET AL. (2005). Tactile estimation of the roughness of gratings yields a graded response in the human brain: An fMRI study. NeuroImage, 25, 90-100.

KlatzKy, R. L., \& Lederman, S. J. (1999). Tactile roughness perception with a rigid link interposed between skin and surface. Perception \& Psychophysics, 61, 591-607.

Klatzky, R. L., Lederman, S. J., Hamilton, C. [L.], Grindley, M., \& Swendsen, R. H. (2003). Feeling textures through a probe: Effects of probe and surface geometry and exploratory factors. Perception \& Psychophysics, 65, 613-631.

Lederman, S. J., Klatzky, R. L., Hamilton, C. L., \& Ramsay, G. I. (1999). Perceiving surface roughness via a rigid probe: Effects of exploration speed and mode of touch. Haptics, 1 (No. 1). Retrieved May 10, 2007, from www.haptics-e.org/Vol_01/index.html.

Lederman, S. J., \& TaYlor, M. M. (1972). Fingertip force, surface geometry, and the perception of roughness by active touch. Perception \& Psychophysics, 12, 401-408.

MadDOX, W. T. (1999). On the dangers of averaging across observers when comparing decision bound models and generalized context models of categorization. Perception \& Psychophysics, 61, 354-374.

MARKS, L. E., \& CAIN, W. S. (1972). Perception of intervals and magni- tudes for three prothetic continua. Journal of Experimental Psychology, 94, 6-17.

McGee, M. R., Gray, P., \& Brewster, S. (2001). Feeling rough: Multimodal perception of virtual roughness. Paper presented at EuroHaptics 2001, Birmingham, U.K.

Moore, T., Broekhoven, M., Lederman, S. [J.], \& Ulug, S. (1991). Q'Hand: A fully automated apparatus for studying haptic processing of spatially distributed inputs. Behavior Research Methods, Instruments, \& Computers, 23, 27-35.

OAkley, I., Adams, A., Brewster, S., \& Gray, P. (2002). Guidelines for the design of haptic widgets. In X. Faulkner, J. Finlay, \& F. Detienne (Eds.), People and computers XVI-Memorable yet invisible (pp. 195211). London: Springer.

Otaduy, M. A., Jain, N., Sud, A., \& Lin, M. C. (2004). Haptic display of interaction between textured models. Paper presented at the IEEE Visualization Conference, Austin, TX.

Petrie, H., O’Neill, A. M., \& Colwell, C. (2002). Computer access by visually impaired people. In A. Kent \& J. G. Williams (Eds.), Encyclopedia of microcomputers (Vol. 28). New York: Dekker.

Reed, C. L., Lederman, S. J., \& Klatzky, R. L. (1990). Haptic integration of planar size with hardness, texture, and planar contour. Canadian Journal of Psychology, 44, 522-545.

SJöström, C. (2000). The IT potential of haptics-Touch access for people with disabilities. Unpublished manuscript, Lund University.

Smith, A. M., Chapman, C. E., Deslandes, M., Langlais, J.-S., \& THIBODEAU, M.-P. (2001). The role of friction and the rate of tangential force change in the subjective scaling of roughness. Paper presented at EuroHaptics 2001, Birmingham, U.K.

Srinivasan, M. A., Basdogan, C., \& Ho, C. H. (1999). Haptic interactions in the real and virtual worlds. In D. J. Duke \& A. Puerta (Eds.), Design, specification and verification of interactive systems 99 (pp. 1-16). New York: Springer.

Stevens, S. S., \& Galanter, E. H. (1957). Ratio scales and category scales for a dozen perceptual continua. Journal of Experimental Psychology, 54, 377-411.

Stevens, S. S., \& Harris, J. R. (1962). The scaling of subjective roughness and smoothness. Journal of Experimental Psychology, 64, 489-494.

Stone, L. A. (1967). Subjective roughness and smoothness for individual judges. Psychonomic Science, 9, 347-348.

Verrillo, R. T., Bolanowski, S. J., \& McGlone, F. P. (1999). Subjective magnitude of tactile roughness. Somatosensory \& Motor Research, 16, 352-360.

WALL, S. A., \& HARWIN, W. S. (2001). Interaction of visual and haptic information in simulated environments: Texture perception. In S. Brewster \& R. Murray-Smith (Eds.), Haptic human-computer interaction (pp. 108-117). Berlin: Springer.

Weisenberger, J. M., Krier, M. J., \& RinKer, M. A. (2000). Judging the orientation of sinusoidal and square-wave virtual gratings presented via 2-DOF and 3-DOF haptic interfaces. Haptics-e, 1 (No. 4). Retrieved January 12, 2006 from www.haptics-e.org/Vol_01/index.html.

Yoshioka, T., Gibb, B., Dorsch, A. K., Hsiao, S. S., \& Johnson, K. O. (2001). Neural coding mechanisms underlying perceived roughness of finely textured surfaces. Journal of Neuroscience, 21, 6905-6916.

\section{NOTES}

1. A full listing of available force feedback devices can be found at the haptics community Web site, haptic.mech.northwestern.edu/ intro/gallery.

2. There are at least four de facto, standard Cartesian coordinate systems in engineering. The convention used in the present software and by SensAble internally in Ghost is the most common mathematical notation. It has a two-dimensional representation in the horizontal plane, like that used on paper ( $x$ right, $y$ away from observer). This may be contrasted with some screen software in which the two-dimensional representation is of a vertical screen ( $x$ right, $y$ vertical). There are also different conventions about whether "away from the observer" is positive or negative.

(Manuscript received August 25, 2005; revision accepted for publication August 21, 2006.) 